\title{
TENSION DES MOTS ET NÉOLOGIE PAR TÉLESCOPAGE DANS LA PUBLICITÉ
}

\author{
Marc BONHOMME \\ Université de Berne
}

\begin{abstract}
This paper shows how lexical telescoping in advertising arouses significant tensions on the language. Some of them are discursive, due on the one hand to the blend of two words that dislocates the integrity of the linguistic sign, and on the other hand to different breaks in the production of advertising statements. Other tensions are pragmatic in that lexical telescoping recategorizes our concepts and reference to the world to win the public acceptance in the direction wished by advertisers. In the end, while illustrating the power of influence of words, lexical telescoping creates an antilexicon in order to make advertising communication more effective.
\end{abstract}

\section{INTRODUCTION}

Parmi les procédés qui contribuent à la tension des mots, la néologie par télescopage, source d'amalgames, occupe une place de choix en français contemporain. Comme le montrent Grésillon (1984) ou Fradin (1997), c'est en effet à notre époque qu'après avoir été l'apanage des expériences littéraires, ce processus atteint son plein développement dans les domaines les plus divers : économie, presse, publicité, etc. Nous nous proposons de faire un rapide bilan sur la néologie par télescopage dans ce dernier domaine, car il en constitue un terrain d'expériences exemplaire. Offrant un corpus homogène et formant un espace langagier des plus productifs, le champ publicitaire révèle on ne peut mieux le cadre global de la créativité par amalgame : celui de la néologie idiolectale, caractérisée par ses tensions sur le lexique. 
Soit l'exemple suivant tiré d'une annonce de La Redoute : (1) «Je suis consommaverti ». Le néologisme consommaverti présente plusieurs traits remarquables :

- Premièrement, une position linguistique floue. Consommaverti est certes une réalité lexicale, consciente et calculée. Mais c'est aussi une réalité syntaxique et même rhétorique. Le Groupe $\mu$ (1970 : 56) voit d'ailleurs dans ce type de mot un « métaplasme par addition ».

- Deuxièmement, une dimension subversive. Un néologisme comme consommaverti perturbe profondément le signe linguistique : violation de l'intégrité du mot ; mise en cause de la séparabilité des signifiants ; dislocation des relations réglées entre le signifiant et le signifié... L'auteur de ce genre de terme se comporte en « lexicoclaste » (Hesbois, $1986: 98$ ) qui convertit le langage en de simples formes malléables à volonté.

À cela s'ajoutent deux autres traits privilégiés dans la néologie par télescopage :

- D'une part, son caractère métalinguistique, en ce que consommaverti se borne à être un nouveau terme créé à partir d'unités lexicales préexistantes, consommateur et averti. Celui-ci consiste donc en un mot sur des mots. Plus exactement, il met en œuvre une relocutivité du lexique, selon laquelle ce dernier est recomposé et réactivé discursivement à un second degré en mots-blocs.

- D'autre part, la néologie par télescopage est soumise à une forte évanescence. Elle est en effet la plupart du temps précaire, ne dépassant pas son cadre énonciatif : consommaverti ne vaut que pour la campagne publicitaire dans laquelle il prend place. De plus, il ne peut être interprété que par un retour en arrière sur les deux mots-sources qui le sous-tendent. Cette réversibilité, qui rappelle le « changement » de Frei (1982:29), singularise consommaverti par rapport à la néologie ordinaire.

Par-delà ces premiers traits, examinons plus en détail les tensions proprement discursives et les tensions pragmatico-référentielles de la néologie publicitaire par télescopage.

\section{TÉLESCOPAGES ET TENSIONS DISCURSIVES}

La néologie par télescopage s'intègre dans la " néologie syntagmatique » de Guilbert (1975 : 101) et plus précisément dans la catégorie des composés discursifs. Cependant, la seule agglutination de signes autonomes qui définit la composition ne saurait suffire pour produire un amalgame. Celui-ci nécessite une interpénétration entre un lexème-support et un lexème-apport au sein d'une 
séquence prédicative. C'est de cette symbiose que l'amalgame tire tous ses effets, notamment en publicité. Or l'intérêt des productions publicitaires est de permettre, par leur variété, de dresser une typologie discursive assez fine des amalgames lexicaux, suivant leurs tensions croissantes sur l'actualisation de la langue.

À un premier degré, le télescopage aboutit à un chevauchement partiel entre deux lexèmes-sources. Celui-ci se manifeste par une troncation au point de rencontre des deux termes concernés. Usuel dans la publicité, ce type constitue la forme classique de la néologie par amalgame et correspond à l'acronymie de Guilbert. On observe soit une apocope du premier lexème : (2) minimaximum (Austin Metro), soit plus rarement une aphérèse du second : (3) Videosaure (Jurassik Park-Universal) ${ }^{1}$. On relève également des mises en dénominateur commun d'un segment homophone appartenant aux deux lexèmes-sources : (4) Bosphoremidable (Jet Tours).

À un deuxième degré, le télescopage se traduit par l'inclusion d'un termeapport dans un terme-support. Le terme-apport reste intact, tandis que le termesupport est perturbé en son centre par cette insertion. On est alors en présence du mot-sandwich de Ferdière (1962). Tantôt le mot-apport se dévoile à travers une manipulation orthographique (graphème $\rightarrow$ trigramme) : (5) ExplEAUsif (Perrier). Tantôt la contamination lexicale apparaît à la suite de la substitution d'un phonème : (6) «Inveskissez en Andorre " (Lop SA). L'inclusion offre des variantes et revêt parfois la forme d'un emboîtement latéral. Ainsi quand elle se déporte vers le début : (7) Teatanic [tea + Titanic ${ }^{2}$ ] (Migros) ou vers la fin du motsupport : (8) Tragicle [tragique + gicle] (Zurich Assurances). Le mot-sandwich se particularise à ce moment en mot-gigogne, terme que nous empruntons à Hesbois (1986) et que nous définissons par l'enchâssement asymétrique d'un mot dans un autre.

À un troisième degré, on assiste à un chevauchement total entre les deux lexèmes à la base du télescopage. Nous parlons dans ce cas de mot-palimpseste (par référence à Genette, 1982), caractérisé par le processus du mot à travers le mot, avec sa mise en volume énonciative. On découvre dès lors une imbrication paronymique, proche du calembour, entre un mot-apport contextuel et un motsupport relégué à l'arrière-plan, mais perceptible dans le signifiant qui l'éclipse : (9) « La plus bleyle rencontre de la semaine» [bel(1)e] (Vêtements Bleyle).

Jusqu'à présent, en dépit de leurs tensions lexicales, les télescopages rencontrés se distinguaient par la hiérarchisation et par l'identification relativement aisée de leurs composantes. La publicité présente très peu de cas d'une dernière catégorie

\footnotetext{
${ }^{1}$ Cet amalgame constitue le titre d'une vidéo sur les dinosaures.

${ }^{2}$ Un tel amalgame forme le slogan d'une annonce vantant l'Ice Tea de la chaîne alimentaire Migros.
} 
de télescopages autrement plus conflictuels : les mots-valises stricto sensu, tels qu'ils ont été pratiqués par Lewis Carroll (1979) et ses imitateurs. Un mot-valise strict comme le fameux slictueux de Carroll se définit par l'éclatement et la fusion étroite de ses composantes (souple + actif + onctueux (?)), par leur absence de hiérarchisation et leur perception aléatoire ${ }^{3}$. D'où son obscurité inhérente et l'important effort interprétatif qu'il nécessite. Soit l'un des rares exemples que nous ayons trouvé en publicité : (10) «Perrier dégrastille le soprano $»^{4}$. Comment lire dégrastille ? S'agit-il d'une forgerie, à savoir un terme spontané sans base lexicale attestée ? S'agit-il d'un parasynthétique idiolectal analysable en dé- + gra $(t) t(e r)+$-ille ? Ou devons-nous lire ici un métissage de lexèmes, auquel cas on a un mot-valise ? Mais sur quels lexèmes s'appuie-t-il ? (dé-)gras + (pé) tille ? (dé-)gras + (dis)tille 5 ? Avec une telle occurrence, le télescopage atteint son degré extrême, celui de l'hybridation totale, instauratrice d'un « monstre lexical » (Bonhomme, 2011 : 11) énigmatique.

La richesse du genre publicitaire en amalgames lexicaux révèle encore d'autres traits tensionnels qui les singularisent. En premier lieu, leurs fréquentes ruptures typographiques affectent la chaîne formelle de l'énoncé: guillemets de distanciation signalant la non-intégration du processus : (11) «Un séjour "formifiant" 》 (Office $\mathrm{du}$ Tourisme de Font-Romeu) ; caractères majuscules mettant en évidence la contamination : (12) JoyEAU (Perrier)... Celle-ci se trouve quelquefois visualisée par une rupture iconique, comme le recours au bichromatisme : (13) Innov[rouge] atique[noir] (Nissan Micra). En outre, des ruptures plus radicales proviennent des recatégorisations des parties du discours opérées par le télescopage, principalement au niveau du lexème-apport : adjectivation du nom ((5) explEAUsif) ou du verbe ((8) tragicle), verbalisation du nom : (14) « Sportez-vous bien » (Volkswagen Golf)... Le résultat en est la création de lexèmes hybrides et hors-norme qui prennent le contrepied des catégories standardisées de la grammaire et qui les réagencent librement dans le déploiement du discours.

Cependant, loin d'être étrangers à toute régulation comme pourraient le suggérer leurs ruptures formelles et catégorielles, les télescopages publicitaires sont canalisés par diverses motivations qui en estompent l'arbitraire apparent. La plus évidente est la motivation phonétique entre les termes amalgamés, souvent connectés à partir d'une analogie dans leurs signifiants, qu'elle se situe au niveau du phonème en (5), de la syllabe en (4) ou à celui de la totalité du mot en (9). Tout

3 Nous suivons ici Chaurand (1977) qui restreint le mot-valise aux télescopages morphologiquement complexes et sémantiquement opaques.

${ }^{4}$ Le soprano désigne le gosier en argot.

${ }^{5}$ Pour une première analyse de cet exemple, voir Adam et Bonhomme (2012). Dans l'ensemble, dégrastille suggère un double sémantisme : suppression d'une négativité + action positive. 
aussi notables sont les motivations syntagmatiques qui justifient par le cotexte l'amalgame effectué. Ces justifications sont de plusieurs ordres : par le nom propre initial de la marque : (15) «FORZA. Forzamusant»; par le cadre géographique de l'énoncé : (16) « À Bourges ça bourge au printemps $»^{6}$; ou par l'anticipation d'un terme-pivot de l'annonce : (14) «Sportez-vous bien! Toujours en pleine forme la Golf coupé sport !». Dans tous ces cas, les télescopages apparaissent comme la condensation d'une situation énonciative plus large qui en fournit la clé interprétative.

\section{Tensions PRAgMATICO-RÉFÉRENTIELLES}

Les brouillages du signifiant qui caractérisent les amalgames publicitaires se prolongent par des tensions pragmatico-référentielles émanant de plusieurs stratégies. Ces tensions portent sur la gestion de la communication médiatique des publicités, de tels amalgames ayant une fonction phatique liée au « besoin d'expressivité » de Frei (1982 : 223). Prenant place dans le slogan, partie accrocheuse par excellence des annonces, ils visent à surprendre le public par des formes étranges, au fort impact visuel et sonore, du type : (4) Bosphoremidable ou (17) « On tabastoppe » (Comité d'éducation pour la santé). Le télescopage avive en cela le contact interactif, tout en renforçant la mémorisation du message transmis. À cette néologie à finalité phatique s'ajoute dans de nombreux cas un effet ludique, fondé sur le plaisir de déstructurer le langage standard. Centrés sur la manipulation du code et sollicitant la fonction poétique du langage au sens de Jakobson (1963), les amalgames lexicaux donnent alors l'illusion d'une gratuité totale, en contradiction avec la finalité commerciale du discours publicitaire.

Par-delà leur tension communicationnelle, les amalgames publicitaires instaurent une tension référentielle sur notre vision du monde, en ce qu'ils recatégorisent nos concepts et notre représentation de la réalité. Cette recatégorisation consiste en divers mixages sémantico-référentiels qui permettent, à partir des syntagmes sous-jacents aux télescopages, de produire une double assertion en même temps (en disant $\mathrm{x}$, on dit aussi y). Exploitant les matrices conceptuelles à la base des énoncés, ces mixages dénotatifs rendent possible une typologie fonctionnelle de la néologie par télescopage. On relève entre autres les syncrétismes sémantico-référentiels suivants :

${ }^{6}$ Référant au festival du Printemps de Bourges, bourge amalgame le nom de cette ville avec le verbe bouge, tout en faisant allusion à bourgeon. 


\section{Matrices qualitatives :}

- Telle entité a telle qualité

(18) Hanimex est extraordinaire

- Telle entité a telle et telle qualité

(13) La Micra est innovante et pratique

- Telle entité a tel prédicat métaphorique

(12) L'eau est un joyau

- Telle qualité concerne telle finalité

(11) Fortifiant pour la forme

\section{Matrices actantielles :}

- Telle action concerne tel produit

(17) Stopper le tabac

- Telle action est dans tel lieu

(19) Etre dorloté au Val d'Aoste

- Telle action a telle cible

(20) Se vacciner contre la grippe

- Telle action a telle finalité

(6) Investir pour le ski

- Tel produit provoque telle action

(21) L'eau fait rêver $>\quad$ Entité-Qualité

Hanimextraordinaire

> Qualité 1-Qualité 2

Innovatique

$>\quad$ Prédicat métaphorique-Entité

JoyEAU

$>\quad$ Finalité-Qualité

Formifiant

$>\quad$ Produit-Action

«On tabastoppe »

$>\quad$ Lieu-Action

« Laissez-vous valdorloter »

$>\quad$ Action-Cible

« Vaxigrippez-vous »

$>\quad$ Action-Finalité

«Inveskissez »

$>\quad$ Produit-Action

FEAUrever

Ces syncrétismes sémantico-référentiels ont au moins trois fonctions :

1. Lexicalisant en un point dénotatif toute une séquence phrastique et donnant naissance à des lexèmes-énoncés, ils apportent le maximum d'informations avec le minimum de substance expressive. Ils répondent ainsi au « besoin d'économie » de Frei (1982: 109), intrinsèque à la publicité qui opère dans des situations d'immédiateté.

2. Par le mécanisme même du figement lexical, tout discursif qu'il soit, ces syncrétismes introduisent une certaine abstraction et une rigidité dans des développements prédicatifs par définition conjoncturels. (20) Se vaxigripper (Institut Mérieux) possède une prégnance et une stabilité sémantique autres que « se vacciner contre la grippe ». Un tel amalgame amplifie la résonance du discours publicitaire et accroît son rendement commercial.

3. Ces syncrétismes fonctionnels participent à une néologie de nature connotative. Il est communément admis que les néologismes interviennent comme une réponse linguistique à une réalité nouvelle, leur lexicalisation dans les dictionnaires garantissant leur nécessité dénominative. Mais il n'en est pas ainsi pour les amalgames publicitaires qui perdent leur pertinence si on les sépare de leur situation énonciative. Prenons (6) «Inveskissez en Andorre » ou (19) « Laissez-vous valdorloter ». Inveskissez et valdorloter 
visent en fait à injecter des valeurs périphériques dans le procès associé au produit promu, bref à le connoter tout en ayant l'air de le dénoter. Le plus souvent, ce surplus connotatif injecté subrepticement par le télescopage dans le mot-support correspond au « concept » ou au message central de l'annonceur. Inveskissez exprime un investissement qui se double ipso facto d'un sémantisme de loisir. Se laisser valdorloter, c'est effectuer au Val d'Aoste un séjour qui ne pourra être qu'euphorique. La réalité dénotée (dépense immobilière, déplacement touristique) se trouve ainsi brouillée par des propriétés imaginaires, quoique envisageables, qui la recatégorisent positivement dans le contexte de l'énoncé. En cela, la majorité des amalgames publicitaires entrent dans le cadre d'une néologie axiologique, orientée à la hausse par le terme-apport. De telles recatégorisations connotatives créent, pour le produit présenté, un positionnement valorisant par rapport à la concurrence.

De surcroît, exerçant une tension argumentative sur le public, non seulement la néologie publicitaire par télescopage bonifie les produits, mais elle a pour objectif d'emporter l'adhésion du lecteur-futur consommateur. Dans ce sens, illustrant le pouvoir d'influence des mots, les brouillages lexicaux et sémanticoréférentiels que l'on a vus aboutissent à différentes stratégies persuasives dont nous ne pouvons que donner un aperçu. Si l'on adopte la terminologie de Perelman et Olbrechts-Tyteca (1988), certains télescopages concentrent un argument basé sur la structure du réel, qu'ils confondent le moyen et la fin (investir et ski dans inveskissez en (6)) ou la cause et la conséquence (l'eau Perrier et le rêve qu'elle est censée susciter dans $f E A U r e v e r$ en $\left.(21)^{7}\right)$. D'autres télescopages condensent un argument qui fonde la structure du réel, principalement par analogie, comme dans l'exemple suivant : (22) «Renault Mégane. Aérodynamite ». D’une part, aérodynamite dissimule l'essentiel d'un raisonnement flou par ressemblance de rapports : l'aérodynamisme (A) est à la Mégane (B) ce que la puissance (C) est à la dynamite (D). D'autre part, ce télescopage transfère le sémantisme d'efficience du phore (focalisé sur la dynamite) à l'intérieur du thème (l'aérodynamisme de la Mégane), donnant à ce dernier une valeur symbolique des plus incitatives pour les automobilistes avides de performances. Sous ses apparences constatives, aérodynamite endosse ainsi une force directive non négligeable. Par ailleurs, la majorité de ces amalgames lexicaux renferment une pétition de principe qui conclut aux qualités du produit sans les démontrer : le Bosphore est d'ores et déjà formidable dans (4) Bosphoremidable; la perfection de Perrier est garantie

\footnotetext{
${ }^{7}$ En plus, $f$ EAUrever évoque l'anglais for ever, « pour toujours », qui donne une durée infinie au rêve.
} 
d'emblée dans (12) JoyEAU. De la sorte, ces amalgames mettent en œuvre une prolepse généralisée dont le but est d'imposer par avance au lecteur la positivité du produit présenté et de court-circuiter toute réserve ou toute contre-argumentation de sa part.

\section{Conclusion}

En conclusion, qu'on l'assimile aux « mots sauvages » de Rheims (1969) ou au « français avancé » de Frei (1982), la néologie publicitaire par télescopage pose le problème des limites du lexique. Que faire en effet de ses réalisations extrêmes et instables qui n'existent que par l'hybridation, qui se détachent difficilement de leur contexte, qui échappent à tout enregistrement lexicographique et qui provoquent de nombreuses tensions dans les énoncés ? Pourtant, ces formes éphémères qui nous ont retenu contiennent quelques leçons. Elles nous rappellent que «c'est dans la parole que se trouve le germe de tous les changements » (Saussure, 1974:138). Elles confirment la vitalité de la néologie discursive, avec ses composantes connotatives et argumentatives. Elles démontrent les insuffisances du lexique standard en face des besoins de la communication, obligée de recourir à une sorte d'antilexique pour trouver sa pleine efficacité. En somme, si l'on se réfère encore à Frei (1982), les amalgames publicitaires constituent un cas-type où la fonction transgresse la norme de la langue pour n'obéir qu'à une seule règle : celle du discours.

\section{Références du corpus}

(1) dans Elle du 07/11/2002 ; (2) dans L'Express du 22/03/2005; (3) dans VSD du 06/10/2004; (4) dans Le Point du 07/08/2014 ; (5) dans L'Hebdo du 17/04/1991 ; (6) dans Paris Match du 02/04/2012 ; (7) dans TV8 du 05/09/1998; (8) dans L'Hebdo du 07/05/2015; (9) dans L'Hebdo du 18/06/2014 ; (10) dans L'Express du 14/10/1985; (11) dans Le Point du 02/07/2008; (12) dans L'Hebdo du 05/03/1991; (13) dans Le Temps du 15/03/2003 ; (14) dans L'Hebdo du 08/05/2011; (15) dans Paris Match du 03/07/2012 ; (16) dans VSD du 18/03/2010 ; (17) dans Le Point du 21/03/2014 ; (18) dans L'Illustré du 14/08/2000 ; (19) dans Le Point du 28/05/2015 ; (20) dans L'Illustré du 14/11/2014; (21) dans L'Hebdo du 21/03/1991; (22) dans Le Point du 14/01/2012.

\section{Références bibliographiques}

ADAM, Jean-Michel, BONHOMME, Marc (2012), L'Argumentation publicitaire, Paris, Armand Colin.

BONHOMME, Marc (2011), « À-peu-près structural et énonciatif dans le mot-valise », Le Français moderne, $\mathrm{n}^{\circ} 79 / 1$, pp. 10-21. 